\title{
TWO DEGREE OF FREEDOM PZT MEMS ACTUATED FLAPPING WINGS WITH INTEGRATED FORCE SENSING
}

\author{
J. S. Pulskamp*, G. L. Smith, R. G. Polcawich, C. M. Kroninger, and E. D. Wetzel \\ US Army Research Laboratory, Adelphi, MD, USA
}

\begin{abstract}
This paper reports on the demonstration of microfabricated two degree of freedom actuated insect-inspired wings for smallscale robotics. Previous efforts demonstrated the feasibility of achieving insect-like stroke amplitudes in thin-film PZT actuated millimeter-scale wings [1]. Lift production in insect flight depends upon unsteady aerodynamic effects associated with threedimensional kinematics utilizing both stroke (i.e. flap) and pitch (i.e. wing rotation) degrees of freedom [2]. Incorporation of wing rotation is essential to mimicking insect wing kinematics and hence force production. Closed loop flight control and device characterization have also motivated the development of feedback sensors. Two degree of freedom PZT MEMS actuators, directly controlling both stroke and pitch, have been monolithically integrated with compliant thin film wings and piezoelectric strain sensors.
\end{abstract}

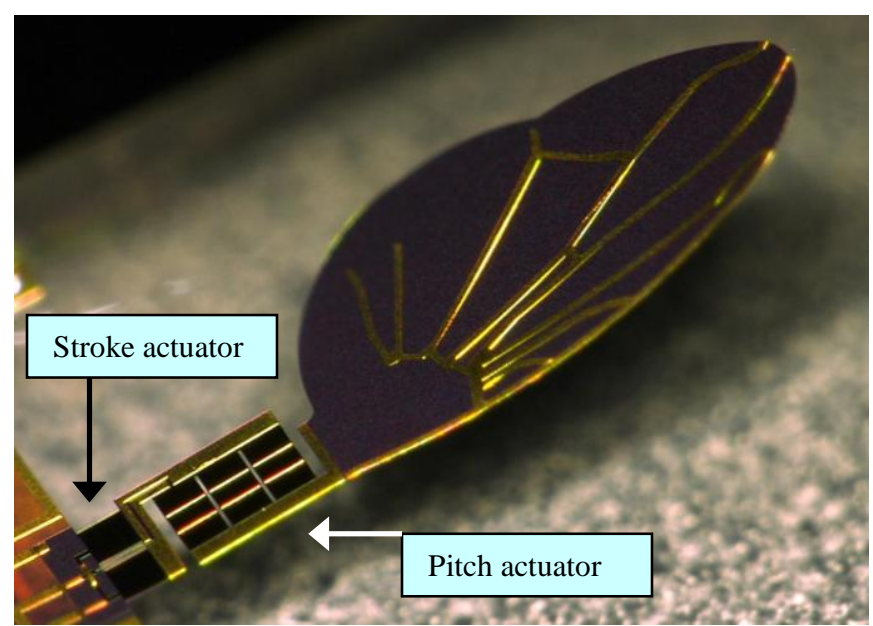

Figure 1: Image of typical design, illustrating PZT MEMS stroke and pitch actuators and thin film wing structure.
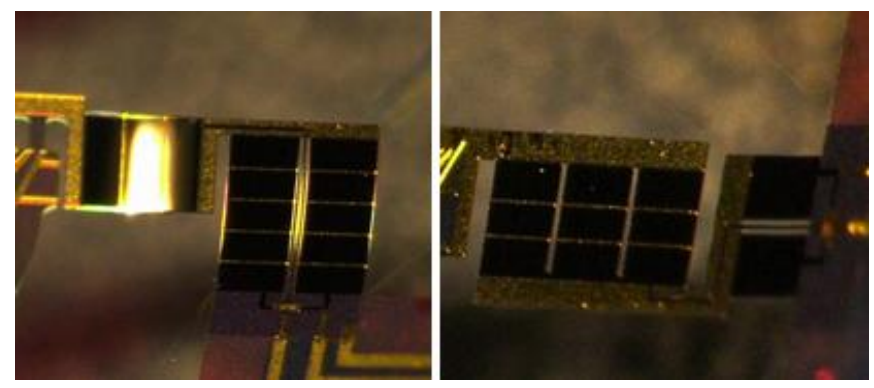

Figure 2: Images of primary designs; left image) the stroke actuator is attached to the wing, right image) the stroke actuator is attached to the substrate.

\section{DEVICE DESIGN}

The primary actuator designs utilize two orthogonal piezoelectric unimorph cantilevered actuators as depicted in Fig. 1. One actuator's plane of bending coincides with the span of the wing while the other is orthogonal; permitting them to drive the stroke and pitch respectively. Two designs were included that exchange the positions of the stroke and pitch actuators (see Fig. 2). A quasi-steady aerodynamic model informed by empirically determined coefficients, capturing various steady and unsteady force components, estimated the expected flight forces in terms of the wing kinematics. Nonlinear analytical models, utilizing these peak aerodynamic loads, provided actuator displacements and stresses for various wing and actuator configurations. This analysis verified that the necessary dimensions of thin film PZT actuators were reasonable to drive these loads at the desired kinematics and provided the unimorph composite thicknesses and actuator dimensions. Finite element analysis was then employed to fine tune the geometries and to determine if the actuator/wing system response times were adequate for the targeted motion (see Fig. 3). Some designs additionally feature passive or actuated hinge structures located just behind the leading edge of the wing to amplify pitch through passive wing rotation or additional actuation. Analytical models were also employed to design piezoelectric strain sensors integrated at the base of the actuator anchored to the substrate with an expected force resolution of a few nano-newtons. These two orthogonal sensors utilize the same piezoelectric layer and bottom electrode as the actuators but feature separate top electrodes. Each sensor is structured as a high aspect ratio (length to width) clamped-clamped structure to ensure the voltage sensitivities of the respective sensors provide information on the dominant orthogonal strains (see Fig. 4).

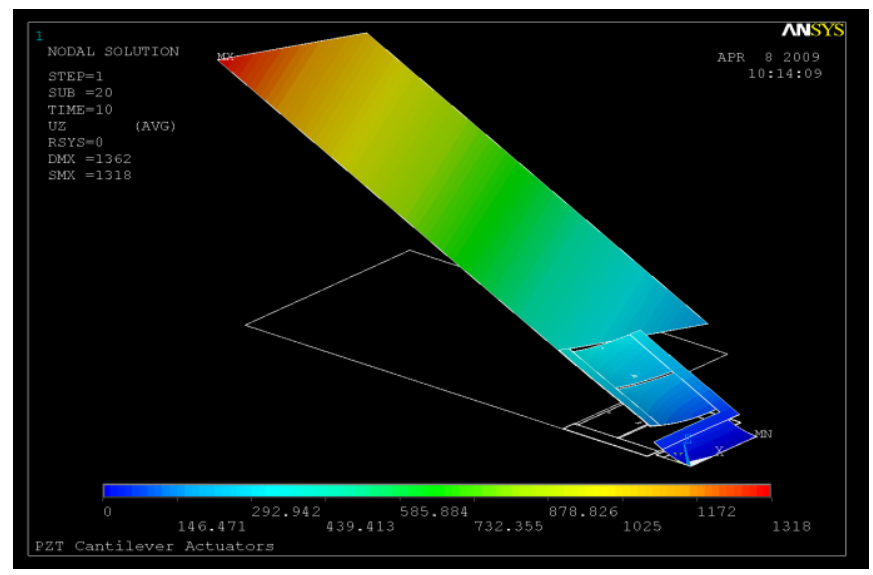

Figure 3: Image of loaded static nonlinear displacement piezoelectric finite element analysis of actuator design and simplified wing structure (precise wing geometry not relevant to analysis).

\section{FABRICATION}

The composite actuators are comprised of silicon dioxidesilicon nitride / titanium-platinum bottom electrode / PZT / top platinum electrode. The composition of the wings vary based on the design but are generally comprised of the same silicon dioxide and nitride layer with either PZT or Ti-Au layers. The venation pattern visible in the images is a thick gold layer. The layer thicknesses used are presented in Table 1. The details of the 
fabrication process are similar to those employed in our earlier work [1].

Table 1:PZT Actuator and wing composite thicknesses. The two silicon dioxide and nitride thicknesses are for different designs and wafers.

\begin{tabular}{|l|c|}
\hline Layer & Thickness $_{(\mathbf{A})}$ \\
\hline Silicon Dioxide/Nitride/Dioxide & 19800 \\
\hline Silicon Dioxide/Nitride/Dioxide $^{2}$ & 10000 \\
\hline Titanium/Platinum & $200 / 850$ \\
\hline PZT & 4970 \\
\hline Top Platinum & 500 \\
\hline Titanium/Gold & $200 / 15000$ \\
\hline Gold & 20000 \\
\hline
\end{tabular}

\section{RESULTS}

The initial characterization has consisted of driving the individual actuators on a probe station with separate signal generators while being monitored with oscilloscopes. A Keyence high speed microscope was used to measure the wing kinematics. The initial results are plotted in Fig. 5 \& 6. In summary, these significantly improved designs have enabled stroke amplitudes in excess of 84 degrees under resonant drive at about 3.4V. Quasistatic displacements of 36 degrees have been measured for pitch.

\section{CONCLUSION}

Our future work will focus on device characterization and addressing proper control. Although lift has yet to be measured with these initial results, the improved designs demonstrate many of the necessary features anticipated to replicate mm-scale flapping insect-inspired flight.

\section{REFERENCES}

[1] J.R. Bronson, et. al., "PZT MEMS Actuated Flapping Wings for Insect-Inspired Robotics", 22nd IEEE International Conference on MEMS, Sorrento, Italy, Jan 2009.

[2] M. H. Dickinson, F. O. Lehmann, and S. P. Sane, "Wing Rotation and the Aerodynamic Basis of Insect Flight", Science, 284, 1954-1960, 1999.

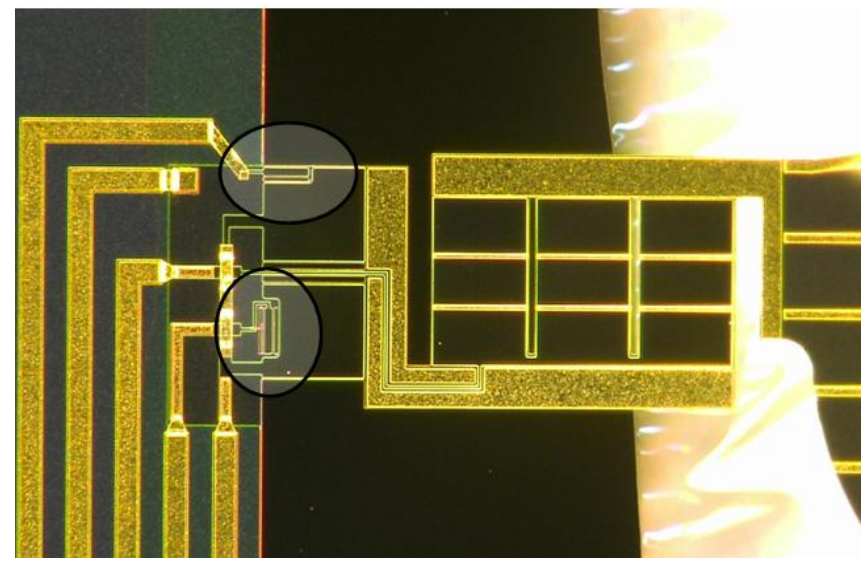

Figure 4: Image device with two integrated piezoelectric strain sensors near the base of the stroke actuator.

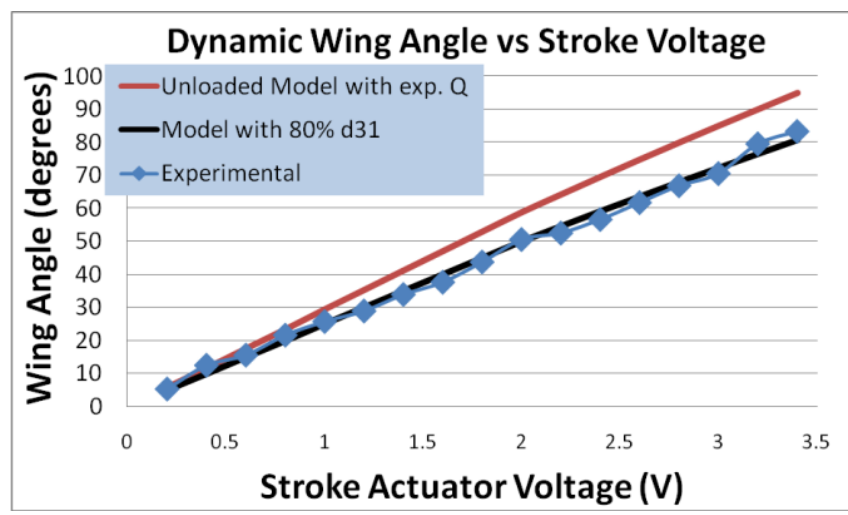

Figure 5: Wing tip angle vs stroke actuator voltage at resonance frequency of $64 \mathrm{~Hz}$. The modeled data utilizes the measured $Q$ (5.9) and typical thin film piezoelectric coefficients for prediction. The model is also presented with a $20 \%$ reduction in $d_{31}$ for improved agreement.

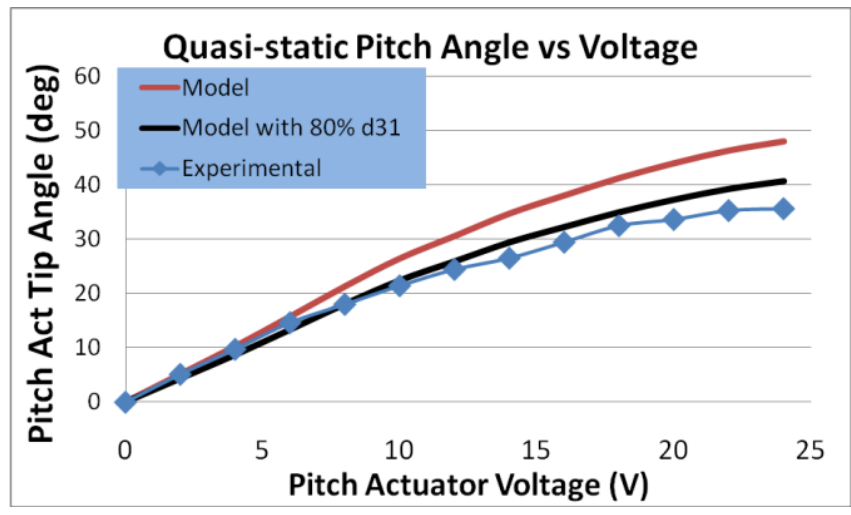

Figure 6: Wing rotation angle vs Pitch actuator voltage at low frequency. The modeled data utilizes typical thin film piezoelectric coefficients for prediction. The model is also presented with a $20 \%$ reduction in $d_{31}$ for improved agreement.

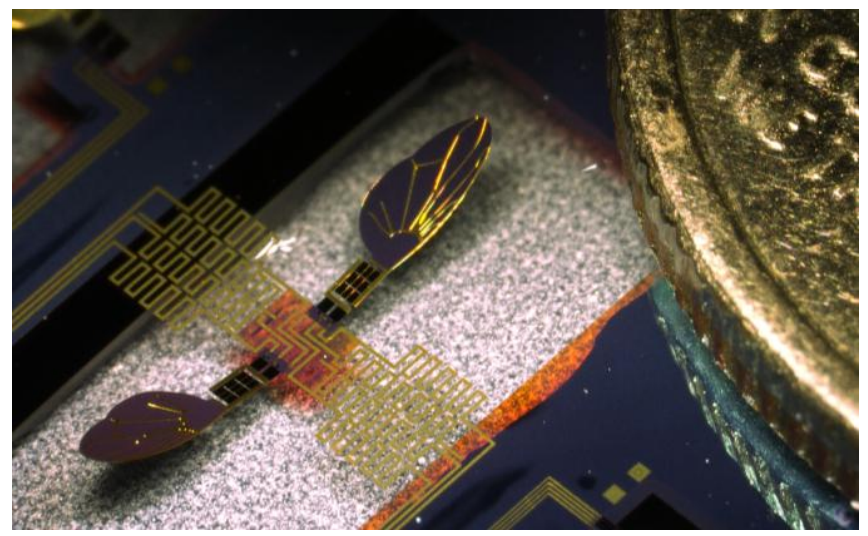

Figure 7: Image of suspended platform with individually controllable wings next to a US dime.

\section{CONTACT}

*J. S. Pulskamp, tel: (301) 394-0016, jeffrey.pulskamp1@us.army.mil. 